\title{
VIOLENCIA HACIA EL PROFESIONAL DE ENFERMERÍA: UNA ETNOGRAFÍA FOCALIZADA
}

\author{
VIOLENCE TOWARDS NURSING PROFESSIONALS: A FOCUSED \\ ETHNOGRAPHY
}

\section{VIOLENNCIA CONTRA O PROFISSIONAL DE ENFERMAGEM: UMA ETNOGRAFIA ESPECÍFICA}

\author{
Claudia Beatriz Enríquez-Hernández* \\ ISRAEL ORTIZ-VARGAS** \\ Ingrid Stephanie Petrovich*** \\ Luis MARTÍNEZ-JiMÉNEZ**** \\ ERnEstina MÉndeZ-Cordero***** \\ Higinio FERnÁNDEZ-SÁNCHEZ******
}

\begin{abstract}
RESUMEN
Objetivo: Describir las experiencias del profesional de enfermería sobre la violencia experimentada en su trabajo. Material y Método: Estudio cualitativo con diseño etnográfico focalizado. Los participantes se seleccionaron utilizando un muestreo no-probabilístico por bola de nieve. Los datos se recolectaron a través de 25 entrevistas a profundidad vía telefónica entre enero y junio de 2018, en tres hospitales de Veracruz, México. El Modelo Interactivo de violencia en el lugar de trabajo diseñado por Chappell y Di Martino fue el referente teórico y la técnica de análisis temática fue con el apoyo del software QUIRKOS. Resultados: Las temáticas resultantes fueron: causas y tipos de agresión, reacción de la víctima ante la agresión y agresor. En su mayoría fueron mujeres (72\%), del turno matutino (52\%), de los servicios de urgencias y medicina interna (28\%). El 68\% refirió no haber denunciado la agresión a sus superiores e identificaron al médico como el principal agresor (44\%), seguido por el familiar del paciente (20\%). Conclusión: Es imperativo la implementación de estrategias
\end{abstract}

\footnotetext{
*Doctora en Ciencias de la Salud en el Trabajo. Facultad de Enfermería región Veracruz, Universidad Veracruzana, Veracruz, México. ORCID: https://orcid.org/0000-0002-4607-0536 E-mail: beenriquez@uv.mx

***octor en Educación. Facultad de Enfermería región Veracruz, Universidad Veracruzana, Veracruz, México. ORCID: https:// orcid.org/0000-0002-4258-9373 E-mail: isrortiz@uv.mx

****Maestra en Enfermería. División de Ciencias de la Salud e Ingenierías Campus Celaya-Salvatierra, Universidad de Guanajuato, Celaya Guanajuato, México. ORCID: https://orcid.org/0000-0001-7220-844X E-mail: is.vasquezventura@ugto.mx

*****Maestro en Enfermería. Facultad de Enfermería región Xalapa, Universidad Veracruzana, Xalapa Veracruz, México. ORCID: https://orcid.org/0000-0002-9326-9177 E-mail: Luis_mtzee2014@hotmail.com

*****Doctora en Educación. Facultad de Enfermería región Veracruz, Universidad Veracruzana, Veracruz, México. ORCID: https://orcid.org/0000-0002-9107-2143 E-mail: ermendez@uv.mx

****** Maestro en Enfermería. Edmonton Clinic Health Academy, Faculty of Nursing, University of Alberta, Alberta, Canada. ORCID: https://orcid.org/0000-0003-4992-7096 E-mail: higinio@ualberta.ca Autor de correspondencia.
} 
para evitar la violencia laboral, así como la formación de pautas a seguir en caso de ser violentada/o.

Palabras clave: Violencia laboral; Enfermeras y Enfermeros; Instituciones de salud; Antropología Cultural; Grupos focales; Investigación cualitativa.

\begin{abstract}
Objective: To describe the experiences of nursing professionals concerning workplace violence. Material and Method: Qualitative study with a focused ethnographic design. Participants were selected using non-probability snowball sampling. Data were collected through 25 in-depth telephone interviews between January and June 2018 in three hospitals of Veracruz, Mexico. The theoretical framework was based on the Interactive Model of workplace violence designed by Chappell and Di Martino and the QUIRKOS software was used for the thematic analysis. Results: Findings alluded to the causes and types of aggression, the victim's reaction to the aggression and the aggressor. Participants were mostly women (72\%), who worked the morning shift (52\%), in the emergency and the internal medicine services (28\%). 68\% indicated not having reported the aggression to their supervisors and identified the physician as the main aggressor (44\%), followed by the patient's family members (20\%). Conclusion: It is critical that hospitals implement strategies to avoid workplace violence, as well as guidelines that nurses and other healthcare professionals can follow in case of violence in the work setting.
\end{abstract}

Key words: Workplace Violence; Nurses; Health Facilities; Cultural Anthropology; Focus Groups; Qualitative Research.

\title{
RESUMO
}

Objetivo: Descrever as vivências do profissional de enfermagem sobre a violência vivenciada em seu trabalho. Material e Método: Estudo qualitativo com desenho etnográfico focado. Os participantes foram selecionados por meio de amostragem náo probabilística em bola de neve. Os dados foram coletados por meio de 25 entrevistas telefônicas em profundidade entre janeiro e junho de 2018, em três hospitais em Veracruz, México. O referencial teórico foi o Modelo Interativo de violência no trabalho desenhado por Chappell e Di Martino e a técnica de análise temática foi realizada com apoio do software QUIRKOS. Resultados: Os tópicos foram: causas e tipos de agressão, reação da vítima frente a agressão e o agressor. A maioria eram mulheres (72\%), do turno da manhã (52\%), dos serviços de emergência e clínica médica (28\%). O 68\% relatou não ter denunciado a agressáo a seus superiores e apontaram o médico como o principal agressor (44\%), seguido do familiar do paciente (20\%). Conclusão: É imprescindível a implementação de estratégias para evitar a violência no trabalho, bem como a formação de diretrizes a serem seguidas em caso de violência.

Palavras-chave: Violência no Trabalho; Enfermeiras e Enfermeiros; Instalaçóes de Saúde; Antropologia Cultural; Grupos Focais; Pesquisa Qualitativa.

Fecha de recepción: 13/03/2021

\section{INTRODUCCIÓN}

La violencia en lugar de trabajo (VLT) se ha convertido en un problema que traspasa límites, entornos y gremios profesionales ${ }^{(1)}$. La VLT, según la Administración de Seguridad y Salud Ocupacional (OSHA), es definida como cualquier acto o amenaza
Fecha de aceptación: 04/05/2021

de violencia física, acoso, intimidación u otras conductas disruptivas amenazantes que ocurran en el lugar de trabajo, que van desde amenazas y abuso verbal hasta agresiones físicas e incluso homicidio ${ }^{(2)}$. En el 2019, la Oficina Internacional del Trabajo (OIT) ha considerado este evento como uno de los riesgos laborales más serios y preocupantes ${ }^{(3)}$, 
asimismo constituye un problema mundial en la salud pública ${ }^{(4)}$.

La evidencia científica existente señala que la violencia laboral, además de asociarse con la agresión física, psicológica o sexual, afecta la dignidad del trabajador, el derecho a no ser discriminado, el respeto de su honra y su integridad física, psíquica y moral ${ }^{(5,6)}$. Por un lado, el riesgo de VLT se presenta con especial importancia en el ámbito sanitario ${ }^{(2,7)}$ debido al ser la primera línea del sistema de salud y actuación en medio del dolor, pérdida y sufrimiento y cuidado en condiciones de salud opuestas a la vida ${ }^{(8-10)}$. Según la encuesta de Bureau en el 2016, se concluye que casi un 70\% del total de incidentes de VLT se producen en el sector salud $^{(7)}$, teniendo un impacto negativo demostrable en la profesión de enfermería ${ }^{(11-13)}$.

Múltiples estudios han demostrado que la VLT, incluyendo otras formas como el acoso e incivilidad, son perpetradas por parte de compañeros de trabajo, supervisores, pacientes o familiares (cuidadores/ acompañantes $)^{(14)}$. De modo que la VLT puede impactar negativamente en la calidad de la atención al paciente ${ }^{(15,16)}$, contribuir al desarrollo de condiciones de salud mental ${ }^{(17,18)}$, reducir el nivel de satisfacción laboral ${ }^{(19-21)}$, incremento en la tasa de agotamiento ${ }^{(22,23)}$ y absentismo ${ }^{(10)}$, además del consumo de drogas ilícitas ${ }^{(24)}$.

A través de la revisión de literatura se detectó que la mayoría de los estudios sobre la VLT han sido para cuantificar el problema, y pocos se han enfocado en describir las experiencias de las víctimas de la VLT en el personal de enfermería en México, de manera que el propósito de este estudio fue describir las experiencias del profesional de enfermería con la violencia en el lugar de trabajo en Veracruz, México.

Esta investigación fue guiada por el Modelo Interactivo de violencia en el lugar de trabajo (MIVLT) propuesto en el año 1998 por Chappell y Di Martino ${ }^{(25)}$. Dicho Modelo permite conocer la VLT desde una lente multifactorial, ya que incorpora diversas variables relacionadas con este fenómeno procedente de la víctima, el agresor y el entorno donde se producen estas acciones ${ }^{(2)}$. El MIVLT permitió estructurar la guía de preguntas y guiar el análisis de los datos. Se retomaron conceptos tales como: factores individuales de riesgo (edad, género y antigüedad), factores laborales de riesgo (contratación, turno, grado académico, servicio), resultados: físicos y psicológicos (tipos de agresión), víctimas (reacción de la víctima ante la agresión) y agresores (paciente, familiar, colega, médico y jefe).

\section{MATERIAL Y MÉTODO}

Diseńo: El presente estudio fue realizado en el 2018 con un diseño cualitativo y se usó un enfoque etnográfico focalizado. Este enfoque se utiliza para investigar grupos poblacionales, posibilitando la observación de la realidad social específica y concreta en un determinado contexto ${ }^{(27,28)}$. Los estudios de investigación etnográfica implican el compromiso de acercarse a la comunidad, de manera de ser aceptados en éstas, para aprender el lenguaje de los participantes, y para comprender los diferentes modos de vida, es decir, sus experiencias ${ }^{(27)}$. Las raíces epistemológicas y ontológicas del enfoque etnográfico focalizado permitieron explorar las experiencias del profesional de enfermería con relación a la violencia en el área de trabajo en tres hospitales públicos del estado de Veracruz, México.

Reflexividad: El primer autor y último autor condujeron las entrevistas. La principal investigadora cuenta con estudios de doctorado en el área de salud laboral y se desempeńa como directora de una Facultad de Enfermería en el Puerto de Veracruz. Este autor reconoce que su alto interés por mejorar las condiciones de trabajo para profesionales de enfermería en el lugar de trabajo pudo haber influido en el análisis de los datos, no obstante, se utilizaron estrategias señaladas más adelante para mitigar el riesgo de sesgo. El último autor es maestro en enfermería, es candidato a doctor en enfermería en Canadá y se desempeña en las áreas de migración, género y salud de la mujer. Todo el equipo de investigación fue capacitado en metodologías cualitativas, así como en análisis de datos cualitativos.

Reclutamiento, muestra y muestreo: Durante el proceso de reclutamiento, el primer y último autor tuvieron un acercamiento con tres profesionales de enfermería que laboran en hospitales de segundo nivel de atención y pertenecían a los colegios de enfermería regionales. Se utilizó un muestreo no-probabilístico por bola de nieve o muestreo dirigido por los participantes ${ }^{(29)}$. Para facilitar el reclutamiento, cada participante recibió 10 trípticos 
en los cuales se establecía el objetivo del estudio, así como información de contacto de los investigadores (ej. teléfono, e-mail). Se indicó que estos trípticos fueran entregados a otros posibles participantes; en caso de estar interesados en participar debían contactar al grupo de investigación.

El tamaño de la muestra se obtuvo hasta conseguir la saturación de los datos. Es decir, se requirió de una investigación exhaustiva de la información obtenida a través de las entrevistas para comprender y entender las implicaciones del fenómeno de interés, a través de las experiencias vividas de los participantes ${ }^{(30,31)}$. La saturación de estos datos se obtuvo a partir del participante número 20, ya que se encontró con repetidas confirmaciones y recurrencia de temas, pero se decidió entrevistar a otros cinco participantes con la finalidad de garantizar el surgimiento de nuevos datos.

Criterios de inclusión y exclusión: Los criterios de selección estaban orientados al personal de enfermería sin distinción de grado académico, de ambos sexos, que hubieran experimentado algún tipo de violencia en su lugar y área de trabajo y quienes aceptaron participar a través de un consentimiento informado verbal. Se excluyeron aquellas personas que no tenían la facilidad de tener la entrevista vía telefónica.

Recolección de datos: La recolección de datos se llevó a cabo a través de entrevistas que se realizaron de manera detallada en un lapso de seis meses, entre enero a junio de 2018. El autor principal junto con el último colega condujeron las entrevistas vía telefónica, las cuales fueron programadas a conveniencia de los participantes. Las llamadas fueron audiograbadas y el tiempo promedio de duración de una entrevista fue de 50 minutos. En cada entrevista se garantizó la protección y confidencialidad de la información personal de cada uno de los participantes, así como el correcto uso de sus datos. Los participantes pudieron expresar libremente sus opiniones, pensamientos y emociones y de esta manera se logró disminuir el sesgo de contexto. La recolección de la información se realizó en dos fases: 1) Datos sociodemográficos de los participantes (ejemplo: edad, sexo) y laborales (ejemplo: años laborales, tipo de contratación), y 2) las experiencias del personal de enfermería con respecto a la violencia en el lugar de trabajo, las personas que ejecutaron la violencia, los motivos percibidos de la violencia, la reacción ante la violencia y las acciones por parte de sus superiores ante la violencia. En caso de que las respuestas de un participante no fueran claras se realizó nuevamente una llamada para realizar preguntas de seguimiento.

Análisis de los datos: La información obtenida en las entrevistas fue transcrita verbatim o textual. Las transcripciones fueron examinadas con las seis fases de análisis de contenido ${ }^{(32)}$. Las fases incluyen: 1) familiarización con los datos obtenidos, 2) generación de categorías o códigos iniciales (palabras, frases, párrafos), 3) búsqueda de temas, 4) revisión de temas, 5) definir temas, y 6) producir el informe final. Para el manejo y gestión de los datos se utilizó QUIRKOS, un software que permite el análisis inductivo de datos cualitativos. El análisis de los datos cuantitativos se realizó mediante la estadística descriptiva, la cual consta de frecuencias y porcentajes, así como medidas de tendencia central: media, mediana y moda. Las validaciones de los datos se hicieron con los participantes y con tres investigadores de este estudio. Una vez que fueron seleccionadas las unidades de análisis, las categorías y subcategorías, se consultó a los participantes para asegurar que los resultados arrojados concordaron con las experiencias previamente relatadas. Esta validación se realizó vía telefónica con los participantes que aceptaron una segunda llamada.

Rigor: Los investigadores aseguraron rigor en el estudio al establecer un apego estricto a la metodología, al realizar una recolección de datos adecuada y al aplicar estrategias óptimas para el análisis de los datos. Las estrategias empleadas incluyeron: el seguimiento de los datos, recopilaciones analíticas con co-investigadores, la comprobación entre los participantes y la recolección de abundantes datos descriptivos.

Ética: Este proyecto de investigación salvaguardó la confidencialidad de los datos de los participantes y se basó en un consentimiento informado verbal, apegándose a la Ley General de Salud en Materia de Investigación para la Salud en México ${ }^{(33)}$ y a la Declaración de Helsinki de la AMMPrincipios Éticos para investigaciones Médicas en Seres Humanos ${ }^{(34)}$. El estudio fue evaluado y aprobado por el Comité de Ética de la Facultad de 
Enfermería de la Universidad Veracruzana, Región Veracruz, respetando la libre decisión a participar y protegiendo el anonimato de los enfermeros. El consentimiento informado verbal señalaba que las llamadas vía telefónica serían audiograbadas y que el material derivado de esta investigación sería utilizado para fines de enseńanza-aprendizaje.

\section{RESULTADOS}

La edad promedio de los participantes fue de 29,52 años $(\mathrm{DE}=46)$. En su mayoría fueron del sexo femenino (725) y el 52\% reportó ser soltero. En cuanto a datos laborales, la mayoría (56\%) tenía una licenciatura, el $24 \%$ una especialidad y el $12 \%$ maestría. El tiempo promedio de antigüedad laboral fue de 5,6 años $(\mathrm{DE}=4,2)$. El 8\% tenía una base laboral, mientras que el resto era personal de contrato. Los servicios donde los participantes se desempeñan eran: Medicina Interna (28\%), Urgencias (28\%), Toco cirugía (20\%), Unidad de cuidados intensivos neonatales (16\%), Pediatría (4\%) y la Unidad de cuidados intensivos (4\%). El $68 \%$ de los participantes reportó no saber el proceso que se lleva a cabo para denunciar una agresión en su lugar de trabajo y el $32 \%$ indicó haber reportado la agresión con sus supervisores.

El análisis cualitativo hizo referencia a cuatro temáticas que surgieron del análisis inductivo y fueron las siguientes: a) Causas de la agresión; b) Tipos de agresión; c) Reacción de la víctima ante la agresión y d) Agresor.

a) Causas de la agresión: En relación a los factores exploratorios de padecimiento, que están estrechamente ligados a pensamientos e ideas subjetivas, los enfermeros revelaron 3 principales causas de agresión:

1. Relaciones interpersonales: Un médico muy soberbio me gritó enfrente de los pacientes haciéndome sentir mal. No dije nada, solo me retiré y segui con mi trabajo (E11).

2. Estructura de poder: Un médico me pidió que subiera a un paciente a piso, no habia camillero y cuando regresó me dijo con voz alta de manera altanera: señorita ya tienen rato que le di la indicación. Le expliqué que no habia llegado aún el camillero, y me contestó que yo estaba ahi para hacer lo que él dijera. No dije más nada, cuando llegó el camillero subi al paciente (E11).

3. Cuidados: Un familiar de un paciente me gritó que nos creíamos, que si tu familiar no era importante, que le podría pasar algo y que lo atendiéramos y ya". Le expliqué que teniamos mucho trabajo y que en cuanto me desocupara lo atendería, siguió gritando, también le gritó al médico (E11).

b) Tipos de agresión: Los enfermeros experimentan violencia de tres formas más frecuentes: física, emocional y sexual, mismas que atentan contra la dignidad y la integridad física, sexual y psicológica de los profesionales de enfermería:

1. Física: Una paciente no quería estar hospitalizada y se comenzó a poner muy agresiva, intenté sujetarla para canalizarla y me pegó en la cara. Quedé sorprendida, no reaccioné, me tomó de sorpresa que hubiese pasado eso (E10).

2. Emocional: Utilizó palabras fuertes, me estuvo acosando, diciéndome que no cuidaba bien a los niños, yo solo contesté lo que habia pasado, pero no fui grosera y siempre fui respetuosa (E2).

3. Sexual: Un médico me pidió mi número de teléfono, me invitó a salir, me agarró el hombro y la cintura. Solo le dije que no estaba interesada y que me respetara. Desde entonces es grosero conmigo en el área de trabajo (E3).

c) Reacción de la víctima ante la agresión: Los enfermeros expresaron diferentes reacciones ante la agresión, tales como: ignorar la situación, reportar el incidente y ataque emocional. Estos datos indican que las experiencias de victimización generan acciones y emociones negativas relevantes:

1. Ignorar la situación: La jefa de enfermería del piso donde laboro me dio a conocer de forma grosera que me habia llegado el contrato pero que ella creía que yo no me merecía el contrato. No dije nada, solo la escuche, acate las órdenes que me dio y me sali (E6).

2. Reportar el incidente: El familiar de un paciente inquieto no aceptó que sujetara al paciente. Le expliqué el motivo. Se molestó más, se levantó y golpeó a mi compañera y a mi en la cara. Lo reportamos y metimos demanda, pero hasta la fecha no nos han resuelto nada, ya pasaron 7 años (E17).

3. Ataque emocional: Una médica cuando se estresa comienza a gritarle a todos, en una guardia me 
comenzó a gritar y me corrió del servicio, y me dijo que le hiciera como pudiera. Comencé a llorar y me retiré (E5).

d) Agresor: Las agresiones físicas, emocionales y sexuales fueron perpetradas por: el médico (44\%), familiar del paciente (20\%), colega (20\%), jefe $(12 \%)$ y paciente $(4 \%)$ :

1. Médico: Un médico me preguntó qué hacía otra compañera, le dije que no sabia y me retire. Cuando regrese me comenzó a gritar que nunca lo volviera a dejar con la palabra en la boca. Le pedi disculpas, aunque dejándole en claro que no, a mi parecer no fui grosera con él (E4).

2. Familiar del paciente: Un familiar de un paciente me gritó que nos creíamos, que si su familiar no era importante, ique le podría pasar algo y que lo atendiéramos ya! Le expliqué que teníamos mucho trabajo y que en cuanto me desocupara lo atendería, siguió gritando, también le gritó al médico. Nos dimos la vuelta y seguimos trabajando (E7).

3. Colega: Cuando iba a aplicar un medicamento una compañera vino y me arrebató la jeringa y me dijo gritando que asi no era. No dije nada solo me quedé callada, realmente me hizo sentir mal (E12).

4. Jefe: Un supervisor me dijo "Pendejo" por una canalización fallida. El intentó y también falló. Le dije entonces también usted es un...P... ;Y me gritó soy tu jefe! Me retiré y lo reporte a un 01800 (E16).

5. Paciente: Un paciente me comenzó a decir: mis ojitos, mi novia, ya cásate conmigo. Lo reporté y me dio mucha pena (E13).

\section{DISCUSIÓN}

En esta investigación, la prevalencia de violencia sufrida por los profesionales de enfermería en sus lugares de trabajo es similar a otros estudios ${ }^{(35-37)}$. A partir de estos estudios, se pueden observar particularidades de la profesión de enfermería en el ámbito social, político, económico y cultural, como la inequidad de género en el desarrollo histórico de la disciplina, idealmente concebida como femenina, lo que podría influir en la falsa creencia de la desventaja y relaciones abusivas en las instituciones de salud durante su jornada laboral. El Consejo Internacional de Enfermería (CIE) recomienda estar alerta y denunciar estos actos; aun más, así se identifica a enfermería como una profesión feminizada.

Los niveles de violencia en contra de los profesionales de enfermería se pueden atribuir a muchos factores, entre otros, que la mayoría de los trabajadores de la profesión sean de sexo femenino. Se sabe que el sexo femenino es identificado generalmente como un grupo vulnerable y susceptible de violencia en todos los estratos sociales y laborales de la sociedad ${ }^{(38-40)}$. Otra causa podría ser el aumento del número de pacientes atendidos ${ }^{(41)}$. La presente investigación demuestra que los profesionales de enfermería sienten que la violencia afecta su desempeño laboral y personal, siendo una de las situaciones más preocupantes la falta de seguimiento en la institución donde se desempeña laboralmente, al no ser lo suficientemente evidenciadas. Siendo los tipos de violencia otro agente causal, la violencia psicológica fue la más reportada por las profesionales, realidad similar a lo reportado por otros autores ${ }^{(37,42)}$, quienes refieren que esta violencia se produce cuando están en el desempeño de sus actividades laborales y se ejerce a través de gritos e insultos.

La violencia física de este estudio, también es reportada en investigaciones afines y los familiares y los compañeros de trabajo fueron los grupos que ejercen esa violencia ${ }^{(36,37,42)}$. Esto es un factor que motiva a los enfermeros a emigrar ${ }^{(43)}$. Lo mismo ocurre con las agresiones de tipo sexual que fueron evidentes en este estudio y similares a otras investigaciones ${ }^{(42,44)}$, donde los perpetradores son varones de mayor rango dentro de la estructura hospitalaria, aunque también se presentan casos de colegas y pacientes. Diferentes estudios corroboran que los pacientes y sus familiares son los agresores más frecuentes de los profesionales de enfermería ${ }^{(45}$, 46) y la violencia ejercida hacia los profesionales de enfermería de esta investigación proviene de pacientes y sus acompañantes. Cabe señalar que los profesionales de la salud que trabajan en salas de emergencia, unidades ambulatorias, unidades de cuidados intensivos, salas de recuperación, anestesia, cuidados intermedios y unidades auxiliares también experimentan violencia con más frecuencia ${ }^{(47)}$.

No obstante, otros estudios han señalado a los colegas, médicos y supervisores como los principales agresores, siendo las estructuras del poder y el cuidado brindado por el profesional los motivos que desencadenan esta violencia. Los resultados de este 
estudio revelan que existen diversas reacciones ante la agresión (ignorar, reportar o ataque emocional) que no se consideran estrategias de afrontamiento, sino actitudes positivas y negativas, asociadas a sentimientos de enojo y frustración ${ }^{(48)}$ porque no existe castigo hacia los agresores, dejando impune esta situación ${ }^{(49-50)}$. Esta violencia física, emocional, psicológica y sexual, tiene graves consecuencias laborales y personales que repercuten en la calidad de la atención, porque reduce los niveles de satisfacción laboral, aumenta el absentismo, el agotamiento y el abuso de sustancias prohibidas ${ }^{(51-53)}$.

A partir de estos resultados se espera que las instituciones de salud de México tomen una serie de medidas para prevenir y erradicar la violencia en ámbito laboral. En particular, que se desarrollen protocolos para facilitar la denuncia por parte de profesionales de salud que experimentan violencia en su lugar de trabajo. Además, que la información de cómo prevenir y denunciar la violencia esté disponible en las áreas de trabajo de todos los profesionales de la salud.

Basado en los resultados obtenidos, es importante señalar la necesidad de mantener políticas públicas en pro de erradicar el abuso de poder por parte de médicos o usuarios. La omisión de los derechos y obligaciones respecto de la violencia en los reglamentos internos de los hospitales o clínicas dejan desamparado al personal de enfermería y se observa la necesidad de implementar evaluaciones que permitan conocer con mayor detalle el impacto de las experiencias tanto a nivel profesional como personal.

De igual forma, se requiere educar a los familiares sobre los procesos de cuidado de enfermería, enfatizando que los servicios de enfermería deben ser aplicados de manera eficiente y oportuna. Los familiares deben entender que los procesos cambian con cada uno de los pacientes que se encuentran a su cuidado y la carga de trabajo que tenga una enfermera no debe ser motivo de malas palabras por parte del familiar.

Esta investigación aporta datos significativos sobre la violencia laboral que experimentan los profesionales de enfermería, sin embargo, tuvo sus limitaciones. Una de ellas fue la falta de triangulación de fuentes de información; otra puede ser que, dada la naturaleza de la investigación cualitativa, los datos no pueden ser generalizados en otros contextos, pero sí pueden ser utilizados como referente para seguir detectando la violencia y sus causas en los ambientes laborales de salud.

\section{CONCLUSIÓN}

Esta investigación nos permitió conocer que al interior de tres hospitales de segundo nivel de atención en Veracruz, México, sí existe violencia hacia el profesional de enfermería y el abordaje cualitativo posibilitó caracterizarla según las causas de la agresión, tipo de agresión, reacción de la víctima ante la agresión y el agresor.

Es necesario documentar los casos de violencia para desarrollar intervenciones en todos los niveles de atención, tanto públicos como privados y se recomienda que desde el ámbito de la investigación se puedan diseñar, implementar y evaluar intervenciones preventivas de la violencia laboral en las instituciones de salud.

\section{REFERENCIAS}

1. World Health Organization (WHO). Framework guidelines for tackling workplace violence in the health sector [Internet]. 2015 [citado 6 sep 2020]. Disponible en: http://www.who.int/violence _injury_prevention/violence/workplace/es/

2. Occupational Safety and Health Administration (OSHA). Workplace Violence in Healthcare [Internet]. 2015 [citado 6 sep 2020]; (800): 10-3. Disponible en: www.osha.gov

3. International Labour Organization (ILO). Framework guidelines for addressing workplace violence in the health sector: The training manual [Internet]. 2005 [citado 6 sep 2020]. Disponible en: https://www.ilo.org/global/topics/safety-andhealth-at-work/resources-library/training/WCMS_ 108542/lang--en/index.htm

4. Li Z, Yan C, Shi L, Mu H, Li X, Li A, et al. Workplace violence against medical staff of Chinese children's hospitals: A cross-sectional study. PLoS One [Internet]. 2017 Jun [citado 6 sep 2020]; 12(6): e0179373. Disponible en: https://dx.plos. org/10.1371/journal.pone.0179373

5. Chávez Porles GA, Román AAM. Violence in Nurses in the workplace [Internet]. Private University Norbert Wiener; 2018 [citado 6 sep 2020]. Disponible en: http://repositorio.uwiener. edu.pe/handle/123456789/463

6. Busnello GF, De Lima TL, Dal Pai D, Brancalione 
D, Calderan MM, Boff BK. Enfrentamiento de la violencia en el trabajo de enfermería en el contexto hospitalario y en la Atención Primaria de Salud. Enf Global [Internet]. 1 Abr 2021 [citado 26 abr 2021]; 20(2): 216-53. Disponible en: https:// revistas.um.es/eglobal/article/view/425181

7. Pierotti D. Workplace Violence. Home Healthc Now [Internet]. 2019 [citado 6 sep 2020]; 37(5): 301-2. Disponible en: http://journals.lww.com/ 01845097-201909000-00014

8. Bordignon M, Monteiro MI. Problemas de salud entre profesionales de enfermería y factores relacionados. Enf Global [Internet]. 29 Jun 2018 [citado 26 abr 2021]; 17(3): 435-69. Disponible en: https://revistas.um.es/eglobal/article/view/302351

9. Molero JMM, Pérez-Fuentes MC, Gázquez LJJ. Workplace harassment among nursing staff. Enfermería Univ [Internet]. 2016 [citado 8 sep 2020]; 13(2): 114-23. Disponible en: https:// www.sciencedirect.com/science/article/pii/ S1665706316000257

10. Chen S, Lin S, Ruan Q, Li H, Wu S. Workplace violence and its effect on burnout and turnover attempt among Chinese medical staff. Arch Environ Occup Heal [Internet]. 2016 [citado 9 sep 2020]; 71(6): 330-7. Disponible en: https:// pubmed.ncbi.nlm.nih.gov/26654585/

11. Phillips JP. Workplace violence against health care workers in the United States. N Engl J Med [Internet]. 2016 [citado 17 sep 2020]; 374: 1661-9. Disponible en: http://www.nejm.org/doi/10.1056/ NEJMra1501998

12. American Nurses Association. Workplace Violence [Internet]. 2019 [citado 17 sep 2020]. Disponible en: https://www.nursingworld.org/practice-policy/ advocacy/state/workplace-violence2/\#

13. Bordignon M, Monteiro MI. Violence in the workplace in Nursing: consequences overview. Rev Bras Enferm [Internet]. 2016 [citado 17 sep 2020]; 69(5): 996-9. Disponible en: https://doi. org/10.1590/0034-7167-2015-0133

14. Alameddine M, Mourad Y, Dimassi H. A national study on nurses' exposure to occupational violence in Lebanon: Prevalence, consequences and associated factors. PLoS One [Internet]. 2015 [citado 16 sep 2020]; 10(9): e0137105. Disponible en:/pmc/articles/PMC4565636/?report=abstract

15. Ilustre Colegio Oficial de Enfermería de Madrid. Protocol against attacks on collegiate/os - CODEM [Internet]. 2019 [citado 5 sep 2020]. Disponible en: https://www.codem.es/protocolo-frente-aagresiones-a-colegiadasos

16. Rathert C, Williams ES, Linhart H. Evidence for the Quadruple Aim. Med Care [Internet]. 2018 [citado 15 sep 2020]; 56(12): 976-84. Disponible en: http://journals.lww.com/00005650-201812000 $-00003$

17. Sun T, Gao L, Li F, Shi Y, Xie F, Wang J, et al. Workplace violence, psychological stress, sleep quality and subjective health in Chinese doctors: A large cross-sectional study [Internet]. 2017 [citado 11 sep 2020]. Disponible en: http://dx.doi. org/10.1136/bmjopen-2017-017182

18. Xu T, Magnusson HLL, Lange T, Starkopf L, Westerlund $\mathrm{H}$, Madsen IEH, et al. Workplace bullying and violence as risk factors for type 2 diabetes: a multicohort study and meta-analysis. Diabetología [Internet]. 2018 [citado 9 sep 2020]; 61(1): 75-83. Disponible en: https://pubmed.ncbi. nlm.nih.gov/29130114/

19. Xu T, Hanson LLM, Lange T, Starkopf L, Westerlund H, Madsen IEH, et al. Workplace bullying and workplace violence as risk factors for cardiovascular disease: A multi-cohort study. Eur Heart J [Internet]. 2019 [citado 9 sep 2020]; 40(14): 1124-34. Disponible en: https://pubmed. ncbi.nlm.nih.gov/30452614/

20. Choi SH, Lee H. Workplace violence against nurses in Korea and its impact on professional quality of life and turnover intention. J Nurs Manag [Internet]. 2017 [citado 9 sep 2020]; 25(7): 508-18. Disponible en: https://pubmed.ncbi.nlm. nih.gov/28547784/

21. Li N, Zhang L, Xiao G, Chen J, Lu Q. The relationship between workplace violence, job satisfaction and turnover intention in emergency nurses. Int Emerg Nurs [Internet]. 2019 [citado 9 sep 2020]; 45: 50-5. Disponible en: https:// pubmed.ncbi.nlm.nih.gov/30797732/

22. Liu W, Zhao S, Shi L, Zhang Z, Liu X, Li L, et al. Workplace violence, job satisfaction, burnout, perceived organisational support and their effects on turnover intention among Chinese nurses in tertiary hospitals: A cross-sectional study. BMJ Open [Internet]. 2018 [citado 8 sep 2020]; 8(6): 19525. Disponible en: http://bmjopen.bmj.com/

23. Zhao SH, Shi Y, Sun ZN, Xie FZ, Wang JH, Zhang SE, et al. Impact of workplace violence against nurses' thriving at work, job satisfaction and turnover intention: A cross-sectional study. J Clin Nurs [Internet]. 2018 [citado 9 sep 2020]; 27(1314): 2620-32. Disponible en: https://pubmed. ncbi.nlm.nih.gov/29446550/

24. Bordignon M, Monteiro MI. Violence in the workplace in Nursing: consequences overview. Rev Bras Enferm [Internet]. 2016 [citado 9 sep 2020]; 69(5): 939-42. Disponible en: http://dx.doi. org/10.1590/0034-7167-2015-0133.

25. Rodríguez VA, Paravic TM. Un modelo para investigar violencia laboral en el sector salud. Rev 
Gaúcha Enferm [Internet]. 2013 [citado 27 abr 2021]; 34(1): 196-200. Disponible en: https://doi. org/10.1590/S1983-14472013000100025

26. Ramacciati N, Ceccagnoli A, Addey B, Lumini E, Rasero L. Violence towards emergency nurses: A narrative review of theories and frameworks. Int Emerg Nurs [Internet]. 2018 Jul [citado 9 sep 2020]; 39: 2-12. Disponible en: 10.1016/j. ienj.2017.08.004.

27. Figueredo BN. La Investigación Cualitativa en Ciencias de la Salud: contribuciones desde la Etnografía. Enfermería (Montevideo) [Internet]. 2017 [citado 27 abr 2021]. Disponible en: http:// dx.doi.org/10.22235/ech.v6iespecial.1445

28. Villar GMG, Mora CMPA, Maldonado RAA. Un acercamiento a la investigación cualitativa en la disciplina del diseño. Rev Iberoam Investig Desarro Educ [Internet]. 2018 [citado 27 abr 2021]; 8(16): 535-56. Disponible en: https://doi.org/10.23913/ ride.v8i16.357

29. Hernández SR, Fernández CC, Baptista LMP. Metodología de la investigación. 6ta. ed. México: McGraw Hill Education; 2014.

30. Faulkner SL, Trotter SP. Data Saturation. In: The International Encyclopedia of Communication Research Methods [Internet]. 2017 [citado 17 sep 2020]. p. 1-2. Disponible en: https://onlinelibrary. wiley.com/doi/abs/10.1002/9781118901731. iecrm0060

31. Fuster GDE. Investigación cualitativa: Método fenomenológico hermenéutico. Propós Represent [Internet]. 2019 [citado 28 abr 2021]; 7(1): 201229. Disponible en: http://dx.doi.org/10.20511/ pyr2019.v7n1.267

32. Díaz-Herrera C. Investigación cualitativa y análisis de contenido temático. Orientación intelectual de revista Universum. Rev gen inf doc [Internet]. 2018 [citado 26 abr 2021]; 28(1): 119-142. Disponible en: https://doi.org/10.5209/RGID.608133

33. López-Pacheco, MC, Pimentel-Hernández, C, Rivas-Mirelles, E., Arredondo-García, JL. Normatividad que rige la investigación clínica en seres humanos y requisitos que debe cumplir un centro de investigación para participar en un estudio clínico en México. Acta pediátr mex [Internet]. 2016 [citado 26 abr 2021]; 37(3): 175-182. Disponible en: https://www.medigraphic.com/pdfs/ actpedmex/apm-2016/apm163f.pdf

34. World Medical Association (WMA). Declaration of Helsinki - Ethical Principles for Human Medical Research [Internet]. 2013 [citado 9 jun 2020]. Disponible en: https://www.wma.net/es/ policies-post/declaracion-de-helsinki-de-la-ammprincipios-eticos-para-las-investigaciones-medicasen-seres-humanos/
35. Dal Pai D, Cristina I, Sturbelle S, Dos Santos C, Tavares JP, Lautert L. Physical and Psychological violence in the workplace of healthcare professionals. Texto Contexto Enfermagem [Internet]. 2018 [citado 9 sep 2020]; 27(1): 2420016. Disponible en: http://dx.doi.org/10.1590/010407072018002420016

36. Zhang L, Wang A, Xie X, Zhou Y, Li J, Yang L, et al. Workplace violence against nurses: A crosssectional study. Int J Nurs Stud [Internet]. 2017 [citado 12 sep 2020]. Disponible en: 10.1016/j. ijnurstu.2017.04.002

37. Oliveira CDS, Martins JT, Galdino MJQ, Perfeito RR. Violence at work in emergency care units: nurses experiences. Rev Lat Am Enfermagem [Internet]. 2020 [citado 6 sep 2020]; 28:e3323. Disponible en: http://dx.doi.org/10.1590/15188345.3856.3323.

38. Najafi F, Fallahi-Khoshknab M, Ahmadi F, Dalvandi A, Rahgozar M. Antecedents and consequences of workplace violence against nurses: A qualitative study. J Clin Nurs [Internet]. 2017 [citado 28 abr 2021]; 27(1-2). Disponible en: $10.1111 /$ jocn. 13884

39. Najafi F, Fallahi-Khoshknab M, Ahmadi F, Dalvandi A, Rahgozar M. Human dignity and professional reputation under threat: Iranian Nurses' experiences of workplace violence. Nurs Heal Sci [Internet]. 2017 [citado 12 sep 2020]. Disponible en: 10.1111/nhs.12297

40. Small MM, Lindo J, Aiken J, Chin C. Lateral violence among nurses at a Jamaican hospital: a mixed methods study. Int $\mathrm{J}$ Adv Nurs Stud [Internet]. 2017 [citado 12 abr 2021]; 6(2): 8591. Disponible en: http://dx.doi.org/10.14419/ ijans.v6i2.8264

41. Roche M, Diers D, Duffield C, Catling-Paull C. Violence Toward Nurses, the Work Environment, and Patient Outcomes. J Nurs Scholarsh [Internet]. 2010 [citado 5 sep 2020]; 42(1): 1322. Disponible en: http://doi.wiley.com/10.1111/ j.1547-5069.2009.01321.x

42. Chimwemwe KB, Mayers P, Duma S. Violence against nurses in the southern region of Malawi. Heal SA Gesondheid [Internet]. 2016 [citado 20 sep 2020]. Disponible en: https://doi.org/10.1016/j. hsag.2016.01.002

43. Fernández-Sánchez $\mathrm{H}$, Enríquez-Hernández CB, Zapíen VM, Horcasitas-Tovar AG. Emigración de profesionales de enfermería en México. Revista Cuidarte [Internet]. 2021 [citado 20 abril 2021]; 12(1): e2008. Disponible en: http://dx.doi. org/10.15649/cuidarte.2008

44. Nurse exposure to physical and nonphysical violence, bullying, and sexual harassment: A 
quantitative review. Int J Nurs Stud [Internet]. 2014 [citado 6 sep 2020]; 51(1): 72-84. Disponible en: www.elsevier.com/ijns\%0AReview

45. Aldrete RMG, Navarro MC, González BR, León CGS, Hidalgo SG. Stress and health in nursing personnel of a tertiary care unit. Revista Cubana de Salud y Trabajo [Internet]. 2017 [citado 15 sep 2020]; 18(1):35-43. Disponible en: https://www. medigraphic.com/pdfs/revcubsaltra/cst-2017/ cst171d.pdf

46. Gazquez JJ, Molero MDM, Pérez-Fuentes MDC, Barragan AB. Quality of life, caregivers and intervention to improve health [Internet]. Madrid: ASUNIVEP; 2018 [citado 12 abr 2019]. Vol II. 641 p. Disponible en: https://ciise.es/files/libros/ BOOK_04.pdf\#page=389

47. Hahn S, Müller M, Hantikainen V, Kok G, Dassen T, Halfens RJG. Risk factors associated with patient and visitor violence in general hospitals: Results of a multiple regression analysis. Int J Nurs Stud [Internet]. 2013 [citado 6 sep 2020]; 50(3): 374-85. Disponible en: https://pubmed.ncbi.nlm. nih.gov/23102404/

48. Shaw DG, Sandy PT. Mental health nurses' attitudes toward self-harm: Curricular implications. Heal SA Gesondheid [Internet]. 2016 [citado 19 sep 2020]; 21(2016):406-414. Disponible en: https://core. ac.uk/download/pdf/82043851.pdf

49. Medina-Maldonado VE, Pozo J, Barrera J, Urgiles P, Ortiz S, Yanez C, et al. Workplace Violence among nursing staff subjective perceptions, preliminary results. Rev cubana enferm [Internet]. 2019 [citado 27 Abr 2021]; 35(3): [aprox. 0 p.] Disponible en: http://www.revenfermeria.sld.cu/index.php/enf/ article/view/2772

50. Alberdi CJ, García GP, Suárez CBJ. Impacto psicosocial en personal sanitario víctima de agresiones por parte de la ciudadanía: una revisión sistemática. Med segur trab [Internet]. 2020 Jun [citado 28 abr 2021]; 66(259): 100-111. Disponible en: https://scielo.isciii.es/pdf/mesetra/ v66n259/0465-546X-mesetra-66-259-100.pdf

51. Davey K, Ravishankar V, Mehta N, Ahluwalia T, Blanchard J, Smith J, et al. A qualitative study of workplace violence among healthcare providers in emergency departments in India. Int J Emerg Med [Internet]. 2020 [citado 6 sep 2020]; 13(1): 33. Disponible en: https://doi.org/10.1186/s12245020-00290-0

52. Havaei F, Astivia OLO, Macphee M. The impact of workplace violence on medical-surgical nurses' health outcome: A moderated mediation model of work environment conditions and burnout using secondary data. Int J Nurs Stud [Internet]. 2020 [citado 28 abr 2021]; 109: 103666. Disponible en: https://doi.org/10.1016/j.ijnurstu.2020.103666

53. Karatuna I, Jönsson S, Muhonen T. Workplace bullying in the nursing profession: A cross-cultural scoping review. Int J Nurs Stud [Internet]. 2020 [citado 28 abr 2021]; 111: 103628. Disponible en: DOI: 10.1016/j.ijnurstu.2020.103628 